\title{
Plenoptic image watermarking to preserve copyright
}

\author{
A. Ansari, A. Dorado, G. Saavedra, M. Martinez Corral \\ Department of optics, University of Valencia, E-46100, Burjassot, Spain \\ Amir.Ansari@uv.es, Adrian.Dorado@uv.es, Genaro.Saavedra@uv.es, Manuel.Martinez@uv.es
}

\begin{abstract}
Common camera loses a huge amount of information obtainable from scene as it does not record the value of individual rays passing a point and it merely keeps the summation of intensities of all the rays passing a point. Plenoptic images can be exploited to provide a 3D representation of the scene and watermarking such images can be helpful to protect the ownership of these images. In this paper we propose a method for watermarking the plenoptic images to achieve this aim. The performance of the proposed method is validated by experimental results and a compromise is held between imperceptibility and robustness.
\end{abstract}

Keywords: Plenoptic Images, Digital Watermarking, DCT, SVD, Robustness, Imperceptibility

\section{INTRODUCTION}

Digital Watermarking is widely used to embed the desired watermark in the host signal. The host signal can be audio, image or video [1]. Some typical examples of application of digital watermarking are copyright protection, avoiding illegal/inappropriate alteration of digital content, medical imaging and error correction [2]. Regarding the domain in which the watermarking algorithm is implemented, the digital watermarking techniques can be divided into spatial domain and frequency domain. It is well-known in the literature that watermarking in frequency domain implies more robustness against common image processing attacks such as noise, compression and filtering [3]. Depending on the watermark embedding mechanism, the embedded watermark can be quite robust against any attack or conversely, highly vulnerable even to the smallest tampering of the host signal. In the literature, the former is often known as robust watermark while the latter is known as fragile one. For ownership protection, the robust watermark is often desirable[4]. The ideal watermarking method is expected to embed as much as information as possible in the host signal such that the watermarked image seems identical to the host iamge and yet, as robust as possible against any kind of attack [5]. A countless number of the papers published in the literature, propose employing wavelet, curvelet, contourlet and Discrete Cosine Transform (DCT) for embedding the watermark [6]. The exploitation of Singular Value Decomposition (SVD) is also addressed in [7].

Contrary to plenoptic cameras, traditional ones record the summation of all the rays passing through each point causing sever loss of the angular information. Plenoptic cameras sample several rays passing through each point delivering much more angular information [8]. Losing the angular information hampers 3D representation of the scene and substantial effort is required to recover the lost angular information. Plenoptic images are widely used in different applications from free view TV to image-based rendering $[9,10]$. The plenoptic images possess a promising feature of focusing after capture [11].

Albeit a tremendous amount of effort has gone to watermark 2D images, to the best of our knowledge the watermarking of plenoptic images is seldom addressed in the literature. In [12] light field rendering is used to generate multiview content and the watermark is embedded in each elemental image separately. The usage of LH and HL subbands of the wavelet transform for light field watermarking is also demonstrated in [13].

In this paper we propose a new method for watermarking of plenoptic images. Our proposed method is based on modification of frequency spectrum of the plenoptic image by DCT and SVD. The remainder of this paper is organized as follows. The proposed method is elaborated in section 2 whereas the experimental results are discussed in section 3 . The conclusion is conducted in section 4. 


\section{THE PROPOSED METHOD}

The captured plenoptic images are commonly represented in RGB domain. For the sake of imperceptibility, the watermark should be embedded in the blue channel which causes the least sensitivity to HVS [14]. Consequently, the proposed method will be applied to blue channel.

To embed the watermark, some microimages of the plenoptic image. In this paper, hereafter the microimage is referred to as $\mu$ I. To redeem the perceptibility of the watermark insertion, $\mu$ Is are selected with appropriate distance. Suppose a binary watermark with the dimensions of $N_{b} \times N_{b}$ is going to be embedded in the host plenoptic image with dimensions of $M \times$ $N_{\mu I} \times M \times N_{\mu I}$ in which $M$ is the length and width of the elemental image, $N_{\mu I}$ is the length and width of the $\mu \mathrm{I}$. It is noticeable that the proposed method works for any arbitrary dimension of the host image and watermark and the assumption of equal length and width is made only for notation convenience.

Each bit of the watermark will be embedded in one $\mu \mathrm{I}$. To achieve this aim, first a DCT transform is applied to the $\mu \mathrm{I}$. The DCT transform of an $M \times N$ matrix is defined as

$$
F(u, v)=\sqrt{\frac{4}{M N}} \sum_{i=0}^{M-1} \sum_{j=0}^{N-1} \Lambda(i) \Lambda(j) \cos \left[\frac{\pi u}{2 M}(2 i+1)\right] \cos \left[\frac{\pi v}{2 N}(2 j+1)\right] f(i, j)
$$

Where

$$
\Lambda(\theta)=\left\{\begin{array}{cc}
1 / \sqrt{2} & \theta=0 \\
1 & O W
\end{array}\right.
$$

Using SVD, then the coefficients of the DCT transform are factorized block by block. Each $M \times N$ matrix can be written as

$$
A_{M \times N}=U_{M \times M} S_{M \times N} V_{N \times N}^{*}
$$

where $U$ and $V^{*}$ are called left and right singular vectors respectively and $\mathrm{S}$ is a diagonal matrix known as singular values. To embed the watermark bit $S(1,1)$ is selected. We have:

$$
S_{w}(1,1)= \begin{cases}S(1,1)-k & w m=0 \\ S(1,1)+k & w m=1\end{cases}
$$

where $S_{w}$ is the matrix of singular values of the watermarked image and all its diagonal elements (except $\left.S_{w}(1,1)\right)$ are identical to $S$ and is the $w m$ watermark bit.

The reference image is later required to extract the watermark. The reference image ref_img carries the values of largest singular value for all the $\mu \mathrm{Is}$. Then the DCT coefficients of the watermarked $\mu \mathrm{I}$ are obtained by

$$
\mu I_{-} w_{D C T}=U * S_{w} * V^{*}
$$

where $\mu I_{-} w_{D C T}$ represents the DCT coefficients of the watermarked $\mu \mathrm{I}$. The watermarked $\mu \mathrm{I}$ in spatial domain is obtained by inverse DCT transform. The inverse DCT transform of an $M \times N$ matrix can be written as

$$
f(i, j)=\sqrt{\frac{4}{M N}} \sum_{u=0}^{M-1} \sum_{v=0}^{N-1} \Lambda(u) \Lambda(v) \cos \left[\frac{\pi u}{2 M}(2 i+1)\right] \cos \left[\frac{\pi v}{2 N}(2 j+1)\right] F(u, v)
$$

$\Lambda(\theta)$ is defined according to eq. (2). This process is repeated until all the watermark bits are embedded.

To extract the watermark, the same $\mu$ Is are selected and DCT and SVD are applied to the $\mu \mathrm{I}$ as mentioned in the embedding procedure. To achieve the extraction process at location $i, j\left(i, j \leq N_{b}\right)$, the corresponding value of the reference image is compared against the largest singular value of the $\mu \mathrm{I}$ :

$$
\operatorname{ext}_{\text {bit }(i, j)}=\left\{\begin{array}{cc}
1 & r e f_{-} i m g(i, j)<s w \\
0 & O W
\end{array}\right.
$$




\section{EXPERIMENTAL RESULTS}

The proposed method is validated by experiment. A plenoptic image is obtained in our lab consisting of $16 \times 16$ elemental images each with spatial resolution of $300 \times 300$. The Peak-Signal-to-Noise-ratio (PSNR) of the watermarked image is defined as:

$$
P S N R=10 \log _{10}\left(\frac{M A X^{2}}{M S E}\right)
$$

where $M A X$ is the maximum value of all the pixels of elemental images and $M S E$ is defined as Mean Square

$$
M S E=\frac{1}{\left(M \times N_{\mu I}\right)^{2}} \sum_{i=0}^{M-1} \sum_{j=0}^{M-1} \sum_{k=0}^{N_{\mu I}} \sum_{l=0}^{N_{\mu I}}(I(i, j, k, l)-W I(i, j, k, l))^{2}
$$

in which Iand WI refer to the host and watermarked image respectively.

The Bit Error Rate (BER) of the extracted watermark is defined as

$$
B E R=\frac{\sum_{i=1}^{N_{b}} \sum_{j=1}^{N_{b}} w(i, j) \bigoplus \widehat{w}(i, j)}{N_{b} \times N_{b}}
$$

in which $w$ and $\widehat{w}$ are the embedded and the extracted watermarks respectively.

Figure 1 shows the central view of the original and watermarked plenoptic image (8th vertical and horizontal view). The embedded and extracted watermark are displayed in figure 2. Figure 3, 4 show the PSNR and BER of the proposed method for different values of the gain factor. As it can be seen from figure 3 and 4, for $k=90$ the PSNR of the watermarked image is more than $57 \mathrm{~dB}$. Please keep in mind that if the watermarked image is not subject to any attack, for any gain factor the embedded watermark can be extracted perfectly with $\mathrm{BER}=0$.
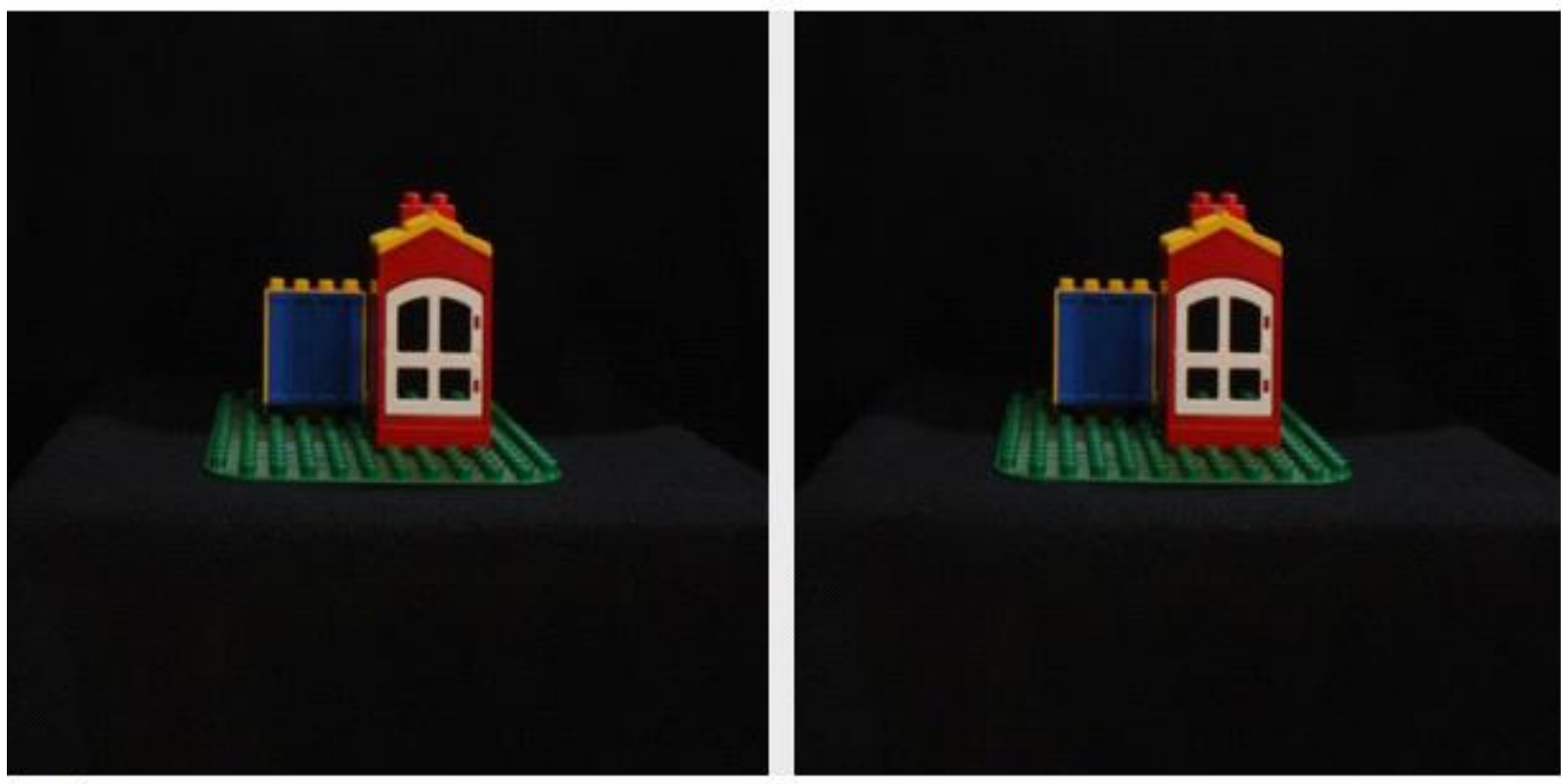

\section{$\mathbf{a} \mathbf{b}$}

Figure 1. central view (8th vertical and horizontal view) of the plenoptic image a) the original image $b$ ) the watermarked image. $k=90$ and PSNR= 57.34dB 

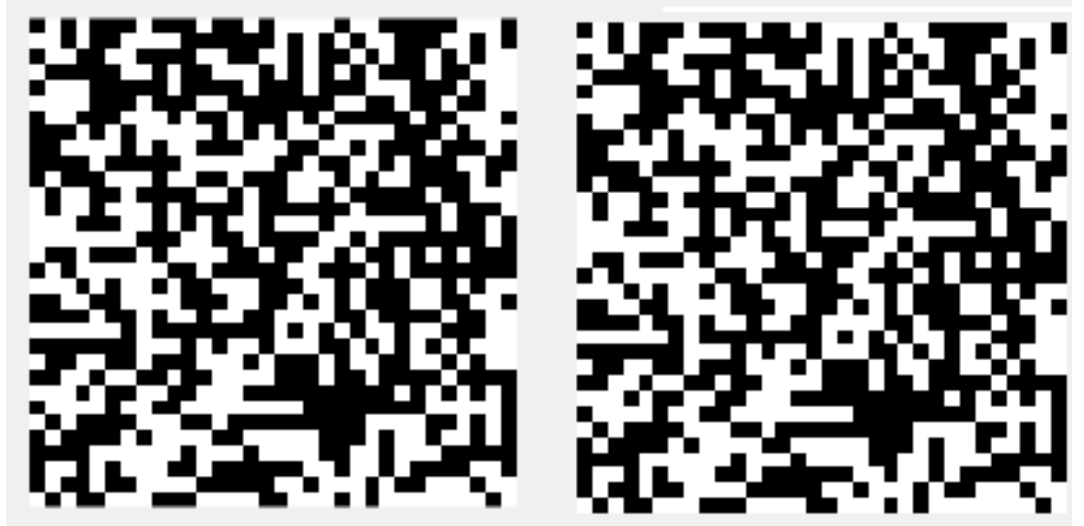

a b

Figure 2. a) embedded logo b) extracted logo $(k=90)$

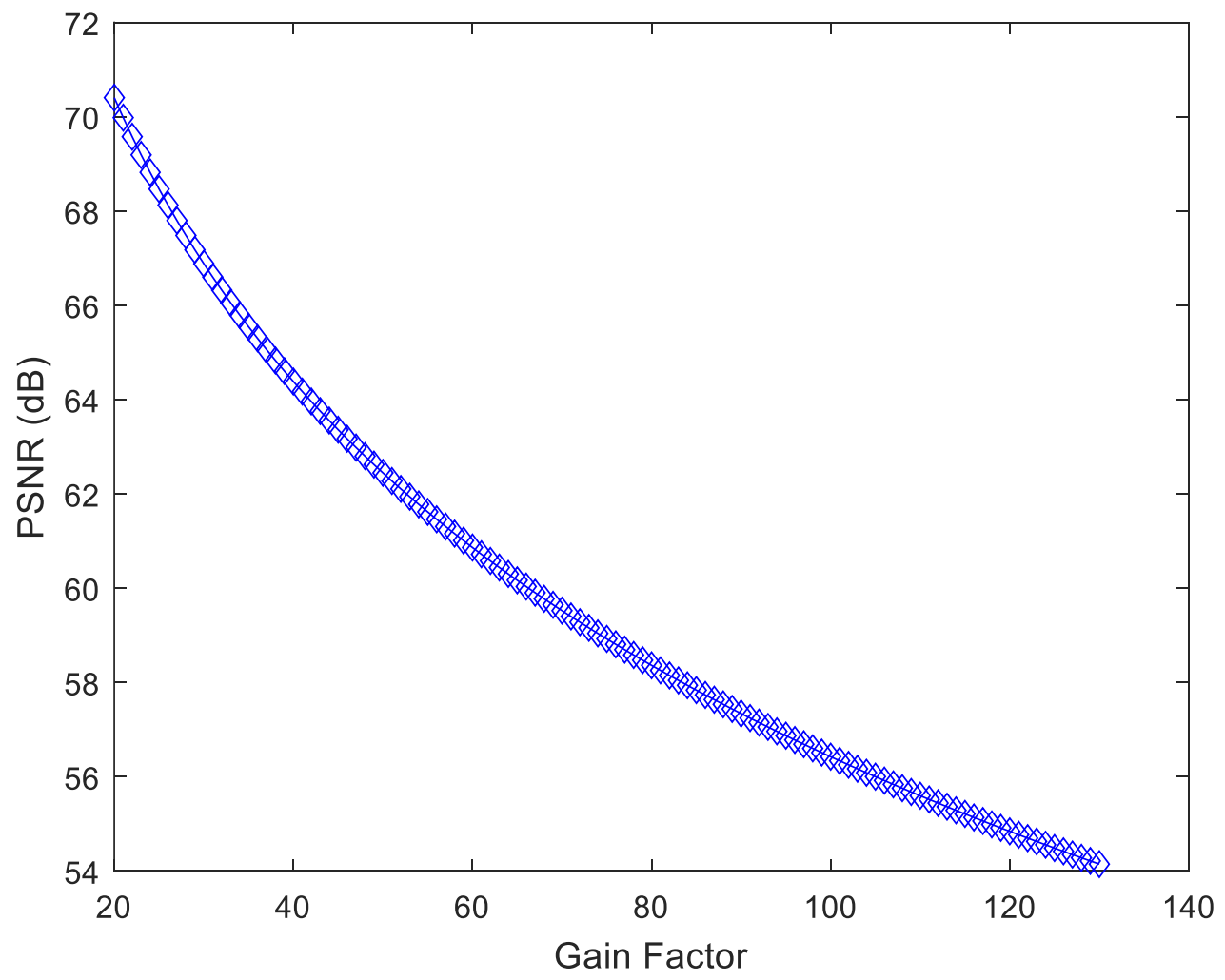

Figure 3. PSNR vs. Gain Factor 


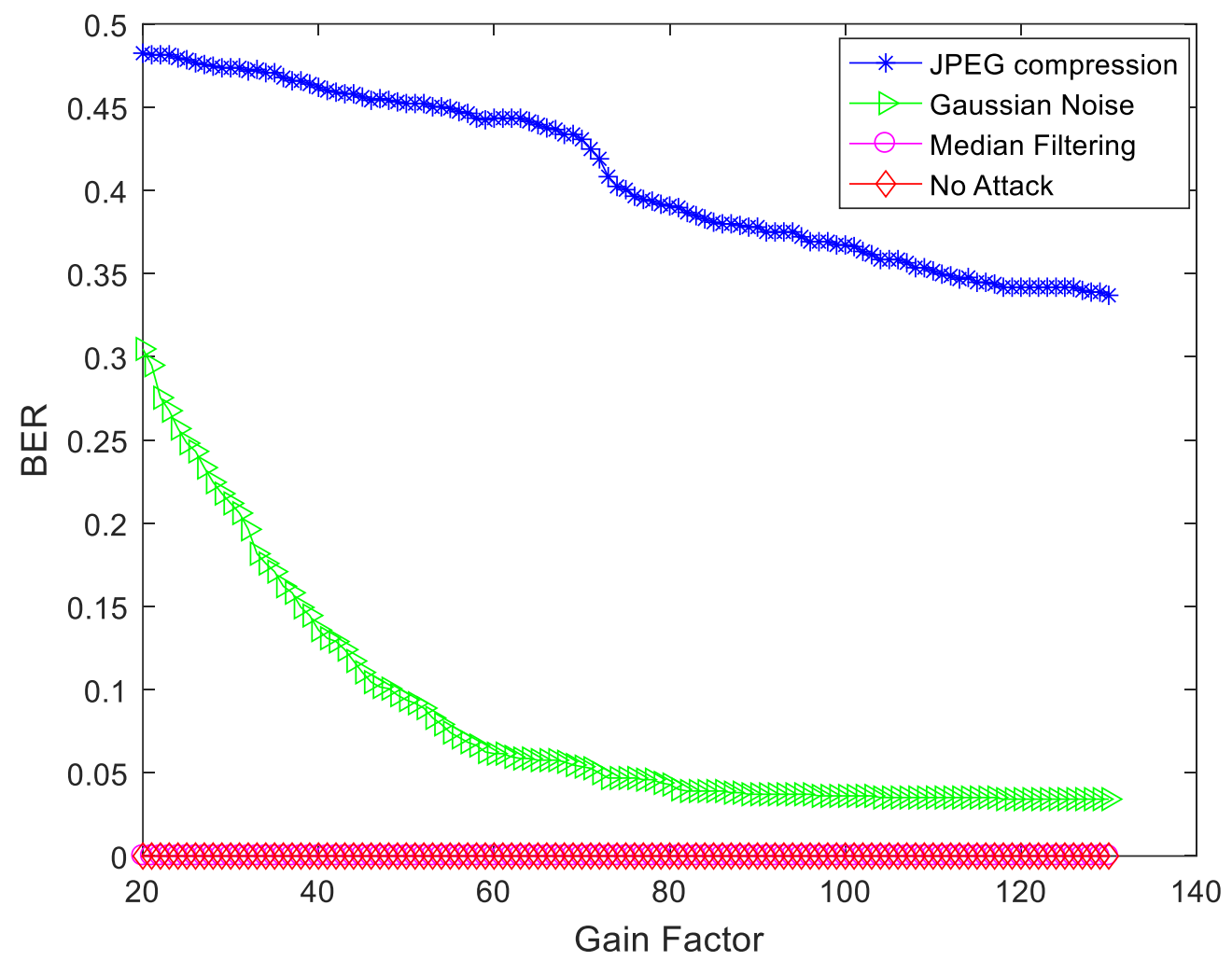

Figure 4. BER for JPEG compression (blue), Gaussian Noise (green), Median Filtering (magenta), No Attack (red)

Plenoptic images may be subject to common image processing attacks. These attacks may take place e.g. in transmission via wireless channels. To assess the robustness of the proposed method against Gaussian noise, the Gaussian noise with variance of 225 is added. It is noticeable that this is an overwhelming noise having strict destructive effect on image content. Figure 4 shows the BER of the extracted watermark from noisy watermarked image.

Another typical attack is JPEG compression. JPEG is widely used and it seems vital for any watermarking system to be robust against JPEG compression. To verify the robustness of the proposed method against JPEG compression, the watermarked image is compressed by JPEG with quality factor of $50 \%$. The results are shown in figure 3 . Please note that the plenoptic image is inherently 4D data and JPEG compression is merely carried out to evaluate the robustness of the proposed watermarking method against typical compression methods.

Other common attack is median filtering. The proposed method exhibits a marvelous robustness against median filtering, such that even for smallest values of gain factor, the proposed method is perfectly robust against median filtering and the embedded watermark can be extracted with zero error. Please be aware that as the proposed method extracts the watermark perfectly and also the BER of median filtering is zero the curve of "No Attack" and "Median Filtering" in figure 4, lay on each other and may seem as a single curve.

\section{CONCLUSION}

In this paper, a new method for plenoptic image watermarking was proposed. The watermarking method utilizes DCT and SVD in both embedding and extraction procedures. The validation of the proposed method is carried out by experimental setup. Additionally, the robustness analysis dignifies the sturdiness of the proposed method against Gaussain noise, JPEG compression and median filtering. 


\section{ACKNOWLEDGMENTS}

This work was supported in by the Plan Nacional I+D+I, under the grant DPI2015-66458-C2-1R, Ministerio de Economía y Competitividad (MINECO), Spain. We also acknowledge the support from the Generalitat Valenciana (GVA), Spain, (grant PROMETEOII/2014/072). A. Dorado acknowledges a predoctoral contract from MINECO. A. Ansari acknowledges a predoctoral contract from EU H2020 program under MSCA grant 676401.

\section{REFERENCES}

[1] C. Busch, W. Funk, and S. Wolthusen, "Digital watermarking: From concepts to real-time video applications," IEEE Computer Graphics and Applications, vol. 19, pp. 25-35, 1999.

[2] G. Boato, V. Conotter, F. G. De Natale, and C. Fontanari, "Watermarking robustness evaluation based on perceptual quality via genetic algorithms," IEEE transactions on information forensics and security, vol. 4, pp. 207-216, 2009.

[3] P. Kumsawat, K. Attakitmongcol, and A. Srikaew, "A new approach for optimization in image watermarking by using genetic algorithms," IEEE Transactions on Signal Processing, vol. 53, pp. 4707-4719, 2005.

[4] C.-J. Cheng, W.-J. Hwang, H.-Y. Zeng, and Y.-C. Lin, "A fragile watermarking algorithm for hologram authentication," IEEE Journal of display technology, vol. 10, pp. 263-271, 2014.

[5] A. Ansari, H. Danyali, and M. Helfroush, "Spread-spectrum robust image watermarking for ownership protection," in 2014 22nd Iranian Conference on Electrical Engineering (ICEE), 2014, pp. 1427-1431.

[6] H. Sadreazami, M. O. Ahmad, and M. S. Swamy, "A study of multiplicative watermark detection in the contourlet domain using alpha-stable distributions," IEEE Transactions on Image Processing, vol. 23, pp. 43484360, 2014.

[7] P. Bao and X. Ma, "Image adaptive watermarking using wavelet domain singular value decomposition," IEEE transactions on circuits and systems for video technology, vol. 15, pp. 96-102, 2005.

[8] E. H. Adelson and J. R. Bergen, "The plenoptic function and the elements of early vision," 1991.

[9] M. N. Do, D. Marchand-Maillet, and M. Vetterli, "On the bandlimitedness of the plenoptic function," in Image Processing, 2005. ICIP 2005. IEEE International Conference on, 2005, pp. III-17.

[10] A. Koz, C. Cigla, and A. A. Alatan, "Free-view watermarking for free-view television," in IEEE International Conference on Image Processing, 2006 2006, pp. 1405-1408.

[11] R. Ng, "Digital light field photography," stanford university, 2006.

[12] A. Koz, C. Cigla, and A. A. Alatan, "Watermarking of free-view video," IEEE Transactions on Image Processing, vol. 19, pp. 1785-1797, 2010.

[13] P. Paudyal, F. Battisti, A. Neri, and M. Carli, "A study of the impact of light fields watermarking on the perceived quality of the refocused data," in 3DTV-Conference: The True Vision-Capture, Transmission and Display of 3D Video (3DTV-CON), 2015, 2015, pp. 1-4.

[14] Y. Wang, J. Ostermann, and Y.-Q. Zhang, "Digital video processing and communications," New Jersy: Prentice Hall, 2001. 